\title{
Opposite and redundant roles of the two Drosophila perilipins in lipid mobilization
}

\author{
Junfeng $\mathrm{Bi}^{1,2, \star}$, Yanhui Xiang ${ }^{1,2, \star}$, Haiyang Chen ${ }^{1,2}$, Zhonghua Liu ${ }^{1}$, Sebastian Grönke ${ }^{3, \ddagger}$, Ronald P. Kühnlein ${ }^{3}$ \\ and Xun Huang ${ }^{1, \S}$ \\ ${ }^{1}$ State Key Laboratory of Molecular Developmental Biology, Institute of Genetics and Developmental Biology, Chinese Academy of Sciences, \\ Beijing 100101, China \\ ${ }^{2}$ Graduate School of the Chinese Academy of Sciences, Beijing 100049, China \\ ${ }^{3}$ Max Planck Institute for Biophysical Chemistry, 37077 Göttingen, Germany \\ ${ }^{*}$ These authors contributed equally to this work \\ ¥Present address: Max Planck Institute for Biology of Ageing, 50931 Cologne, Germany \\ ${ }^{\S}$ Author for correspondence (xhuang@genetics.ac.cn) \\ Accepted 27 March 2012 \\ Journal of Cell Science 125, 3568-3577 \\ (c) 2012. Published by The Company of Biologists Ltd \\ doi: $10.1242 / j c s .101329$
}

\section{Summary}

Lipid droplets are the main lipid storage sites in cells. Lipid droplet homeostasis is regulated by the surface accessibility of lipases. Mammalian adipose triglyceride lipase (ATGL) and hormone-sensitive lipase (HSL) are two key lipases for basal and stimulated lipolysis, respectively. Perilipins, the best known lipid droplet surface proteins, can either recruit lipases or prevent the access of lipases to lipid droplets. Mammals have five perilipin proteins, which often exhibit redundant functions, precluding the analysis of the exact role of individual perilipins in vivo. Drosophila have only two perilipins, PLIN1/LSD-1 and PLIN2/LSD-2. Previous studies revealed that PLIN2 is important for protecting lipid droplets from lipolysis mediated by Brummer (BMM), the Drosophila homolog of ATGL. In this study, we report the functional analysis of PLIN1 and Drosophila HSL. Loss-of-function and overexpression studies reveal that unlike PLIN2, PLIN1 probably facilitates lipid mobilization. HSL is recruited from the cytosol to the surface of lipid droplets under starved conditions and PLIN1 is necessary for the starved induced lipid droplet localization of HSL. Moreover, phenotypic analysis of plin1;plin2 double mutants revealed that PLIN1 and PLIN2 might have redundant functions in protecting lipid droplets from lipolysis. Therefore, the two Drosophila perilipins have both opposite and redundant roles. Domain swapping and deletion analyses indicate that the C-terminal region of PLIN1 confers functional specificity to PLIN1. Our study highlights the complex roles of Drosophila perilipin proteins and the evolutionarily conserved regulation of HSL translocation by perilipins.

Key words: PLIN1, PLIN2, HSL, Lipid droplet, Lipolysis

\section{Introduction}

Proper lipid storage is vital for maintaining energy homeostasis in most, if not all, eukaryotic species. Understanding the molecular mechanisms of lipid storage regulation is critical to prevent the rapid growth of fat storage disorders such as obesity in humans (Flier, 2004; Gesta et al., 2007). The lipid droplet, long thought of as an inert organelle, is important not only as the main lipid storage site inside cells but also for metabolic regulation (Farese and Walther, 2009; Martin and Parton, 2006). Many cellular events, including autophagy and SNARE-dependent intracellular vesicle transport, affect lipid storage in lipid droplets (Boström et al., 2007; Singh et al., 2009). Ultimately, the homeostasis of lipid storage and the dynamics of lipid droplets are controlled by the balance of lipolysis and lipogenesis (Thiele and Spandl, 2008). Lipolytic regulation of lipid droplets mainly occurs on the surface of lipid droplets through the accessibility of various lipases. Under nutrient-poor conditions, more lipases are recruited to lipid droplets to release more fatty acids from triacylglycerol (TAG) (Walther and Farese, 2009; Zimmermann et al., 2009). However, the fine regulation of lipid droplet dynamics under physiological and pathological conditions is still not fully defined.
Lipid droplets contain a lipid core surrounded by a lipid monolayer that is coated with proteins, the best known of which are PAT (perilipin, ADRP and TIP47) domain proteins, collectively named perilipins (Kimmel et al., 2010). Perilipins dynamically interact with lipases and influence their association with lipid droplets to regulate lipid homeostasis. The most intensively studied member of the family, Perilipin1, serves opposite roles in the regulation of basal and hormone-stimulated lipolysis. Under basal conditions, Perilipin1 prevents lipases from accessing lipid droplets. During times of energy deficit, Perilipin1 is phosphorylated by PKA in response to hormonal signals. Phosphorylated Perilipin1 facilitates maximal lipolysis by recruiting hormone-sensitive lipase (HSL) and allowing adipose triglyceride lipase (ATGL) to access the lipid droplet (Granneman et al., 2009; Miyoshi et al., 2006; Sztalryd et al., 2003; Zimmermann et al., 2004). Plin1 mutant mice exhibit a lean phenotype and Plin 1 deficiency reverses the obesity in mice with a mutation in Lepr, the leptin receptor gene. These phenotypes are derived from loss of the anti-lipase protective effect of Perilipin1 under normal conditions (Martinez-Botas et al., 2000; Tansey et al., 2001). Genetic variations in the PLIN1 gene have been associated with metabolic disorders in humans, 
including type-2 diabetes and partial lipodystrophy (Bickel et al., 2009; Gandotra et al., 2011).

Besides Perilipin1, which has at least three isoforms, four other Perilipins have been found in mammals, namely ADRP/ Perilipin2, TIP47/Perilipin3, S3-12/Perilipin4 and OXPAT/ Perilipin5 (Bickel et al., 2009). Interestingly, at different stages of lipid droplet biogenesis, these proteins localize differently on the surface of lipid droplets, indicating their functional specificity (Wolins et al., 2005). Moreover, both ADRP and TIP47 are involved in lipid droplet biogenesis and exhibit redundant functions with perilipin in antagonizing lipolysis (Bulankina et al., 2009). Relatively little is known about S3-12 and OXPAT. The complexity of perilipins raises many questions. Why do cells need more than one perilipin? What is the exact function of individual perilipin in vivo? Are there functional differences among perilipins? If so, what determines the functional differences? A genetically amenable organism with fewer perilipins would be useful to address these questions.

Perilipins are evolutionarily conserved from Drosophila to humans. Importantly, compared to mammals, Drosophila has only two perilipins, PLIN1/LSD-1 and PLIN2/LSD-2 (Bickel et al., 2009). Drosophila shares most of the same basic metabolic functions and lipid metabolism-related genes with higher animals (Baker and Thummel, 2007; Schlegel and Stainier, 2007). For instance, the insulin pathway, which regulates lipid and carbohydrate metabolism, is highly conserved in Drosophila (Baker and Thummel, 2007; Barbieri et al., 2003). In addition, many of the organ systems that control lipid uptake, storage, and metabolism in humans are present in Drosophila. The Drosophila fat body carries out the fat storage function of adipose tissue and also has a liver-like function due to its detoxification roles. Besides the fat body, Drosophila also has specialized hepatocytelike cells called oenocytes, which are important for the regulation of lipid mobilization (Gutierrez et al., 2007). Therefore, it is not surprising that Drosophila has emerged as an important model system to study conserved mechanisms of lipid metabolism (Baker and Thummel, 2007; Pospisilik et al., 2010). For example, two independent RNAi screens using Drosophila cell lines recently revealed that the vesicular transport coatomer protein I (COPI) complex is required for lipid droplet morphology and lipid storage (Beller et al., 2008; Guo et al., 2008). Another genome-wide RNAi screen identified hedgehog as an important fat storage regulator in both flies and mice (Pospisilik et al., 2010).

The ease of genetic manipulation in Drosophila has greatly facilitated functional studies of PLIN1 and PLIN2. Studies of Drosophila plin2 mutants revealed that PLIN2 is required for lipid droplet transport in Drosophila embryos and is important for protecting lipid droplets from lipolysis (Fauny et al., 2005; Grönke et al., 2003; Welte et al., 2005). Drosophila counterparts of the two mammalian lipases, ATGL and HSL, are also present. Mutations of bmm, the Drosophila homolog of ATGL, lead to obese flies, while $\mathrm{bmm}$ overexpression depletes stored lipids (Grönke et al., 2005). A further study showed that plin2 genetically antagonizes the activity of $\mathrm{bmm}$ (Grönke et al., 2005). Drosophila HSL (dHSL), however, has not yet been studied. A recent report showed that PLIN1 is important for stimulated lipolysis and may also have a structural role in lipid droplets (Beller et al., 2010). plin1 mutants exhibit adult-onset obesity. plin1 mutant fat cells have large lipid droplets and have more BMM localized to lipid droplets. These findings shed more light on the function of PAT domain proteins in Drosophila and raise more intriguing questions. How is PLIN1 involved in lipolysis? How can the increased localization of BMM to lipid droplets explain the large lipid droplet phenotype? What is the role of dHSL in lipolysis? What determines the functional differences between PLIN1 and PLIN2? We addressed these questions through genetic and functional analysis of Drosophila PLIN1, PLIN2 and dHSL. Our study reveals both opposite and redundant roles of PLIN1 and PLIN2 in lipid mobilization. In addition, we found that PLIN1 may facilitate lipid mobilization by allowing dHSL to localize to lipid droplets.

\section{Results}

\section{PLIN1 and PLIN2 exhibit different functions and localizations}

We initially compared the loss-of-function phenotype of the two Drosophila perilipin genes, plin1 and plin2, in the larval fat body. Consistent with previous reports (Beller et al., 2010; Grönke et al., 2003), plin2 $2^{K G 00149}$ mutants have smaller lipid droplets than the wild type (supplementary material Fig. S1). In contrast, plin1 RNAi driven by the fat body-specific ppl-Gal4 resulted in large lipid droplets (supplementary material Fig. S1), implying that PLIN1 and PLIN2 have opposite functions. To further explore this possibility, we analyzed the overexpression phenotypes of plin1 and plin2. Overexpression of plin1 with a strong ubiquitous $t u b-G a l 4$ or fat-body-specific ppl-Gal4 resulted in small lipid droplets (supplementary material Fig. S1 and data not shown). On the other hand, overexpression of plin2 slightly increased the size of lipid droplets, consistent with its role in protecting lipid droplets from lipolysis (supplementary material Fig. S1). Together, these data suggest that plin1 and plin2 likely have opposite functions.

To define the specific cellular roles of PLIN1 and PLIN2, we examined their localization patterns using fluorescent proteintagged transgenes. Tagged PLIN1 and PLIN2 fully rescued the plin1 and plin2 mutant larval lipid droplet phenotypes respectively, indicating that the fusion proteins are functional and likely reflect the in vivo protein localization pattern (supplementary material Fig. S2). We found that PLIN1mCherry was localized on the surface of most, if not all, lipid droplets (Fig. 1A). PLIN2-EYFP was mainly localized on the surface of small lipid droplets close to the nuclei or at the

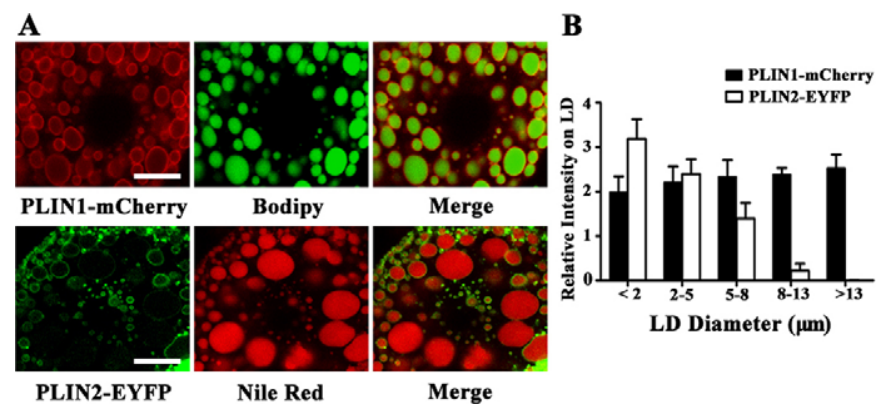

Fig. 1. PLIN1 and PLIN2 have different localizations. (A) Fluorescent images of wandering stage third-instar larval fat bodies. The fluorescent proteins are expressed with tub-Gal4. Bodipy or Nile Red stains lipid droplets. PLIN1-mCherry (red) is found on most, if not all, lipid droplets; PLIN2-EYFP (green) intensity is high on small lipid droplets and low on large lipid droplets. Scale bars: $20 \mu \mathrm{m}$. (B) The relative intensity of PLIN1mCherry and PLIN2-EYFP on lipid droplets of different sizes. 
periphery of the cells (Fig. 1A). Interestingly, as the lipid droplet size increased, the lipid droplet surface level of PLIN2-EYFP decreased while the PLIN1-mCherry level remained unchanged (Fig. 1B). This phenomenon is reminiscent of the lipid droplet localization of PAT domain proteins in mammals, in which ADRP, TIP47, and S3-12 are localized to small lipid droplets while Perilipin1 is localized to large lipid droplets (Wolins et al., 2005). The differences in fusion-protein localization further support the conclusion that PLIN1 and PLIN2 have distinct roles.

\section{plin1 promotes lipid mobilization}

To confirm the plin1 RNAi phenotype, we turned to plin1 mutants. Transposon line MB10034 has a Minos transposon inserted into the $5^{\prime}$-UTR of plin1 (supplementary material Fig. S3). Using MB10034, we generated several plin1 mutants by transposon imprecise excision. plin $1^{38}$ retains a $\sim 2 \mathrm{~kb}$ transposon fragment and has a perfect deletion of intron 3, exon 4 and intron 4 of plin1-RA transcript, which encodes the longest protein isoform of PLIN1 (supplementary material Fig. S3). It is not known how imprecise Minos transposon excision leads to this deletion $1 \mathrm{~kb}$ away from the transposon insertion site. Instead of affecting the coding region, $\operatorname{plin} 1^{110}$ and $\operatorname{plin} 1^{150}$ retain $\sim 4 \mathrm{~kb}$ and $\sim 1 \mathrm{~kb}$ transposon fragments, respectively. Based on RT-PCR results, $\operatorname{plin} 1^{38}$ is likely a strong loss-of-function or null allele of plin1, while plin $1^{110}$ and plin $1^{150}$ are hypomorphic alleles (supplementary material Fig. S3). Consistent with the RNAi results, plin1 mutant larval fat bodies have large lipid droplets and the phenotype is stronger in plin $1^{38}$ null mutants than the hypomorphic mutants (Fig. 2A; supplementary material Fig. S3). Interestingly, compared to the wild type, the total level of glyceride in wandering stage third-instar larvae is not increased in $p$ lin $1^{38}$ mutants, even though they have large lipid droplets (Fig. 2B). These results are consistent with a previous report that plin1 mutants have large lipid droplets and exhibit obesity only at the adult stage (Beller et al., 2010).

We then examined lipid droplets in the larval fat body under starvation, a condition that promotes lipid mobilization. During starvation, lipids are released from lipid droplets by lipolysis. Wild-type animals have small lipid droplets and reduced levels of total glyceride under starved conditions (Fig. 2B,C). In contrast, under starved conditions, both plin1 RNAi and plin $1^{38}$ mutants contain larger lipid droplets than the wild type (Fig. 2C). Moreover, the level of total glyceride in $\operatorname{plin} 1^{38}$ mutants is barely decreased under starvation compared to fed conditions, and is significantly higher than that of starved wild-type animals (Fig. 2B). We also examined the levels of circulating lipids under fed and starved conditions. Stimulated lipolysis in the fat body results in high levels of circulating lipids. Larval oenocytes, which are specialized cells of the larval epidermis, monitor the levels of circulating lipids (Gutierrez et al., 2007). Under fed conditions, oenocytes of the wild type, ppl-GAL4/+ or UAS-plin1/+ animals stain weakly with Oil Red O because the levels of circulating lipids are low and less TAG biosynthesis occurs in oenocytes. In contrast, under starved conditions, stimulated lipolysis results in high levels of circulating lipids and subsequently causes strong Oil Red O staining in oenocytes (Fig. 2D). In plin1 RNAi flies and in plin $1^{38}$ mutants, the intensity of Oil Red O staining in oenocytes is very weak under fed conditions (Fig. 2D). However, under starved conditions, the staining intensity is greatly reduced compared to that of control (Fig. 2D). Together, these results demonstrate that plin1 mutants are defective in lipid mobilization.

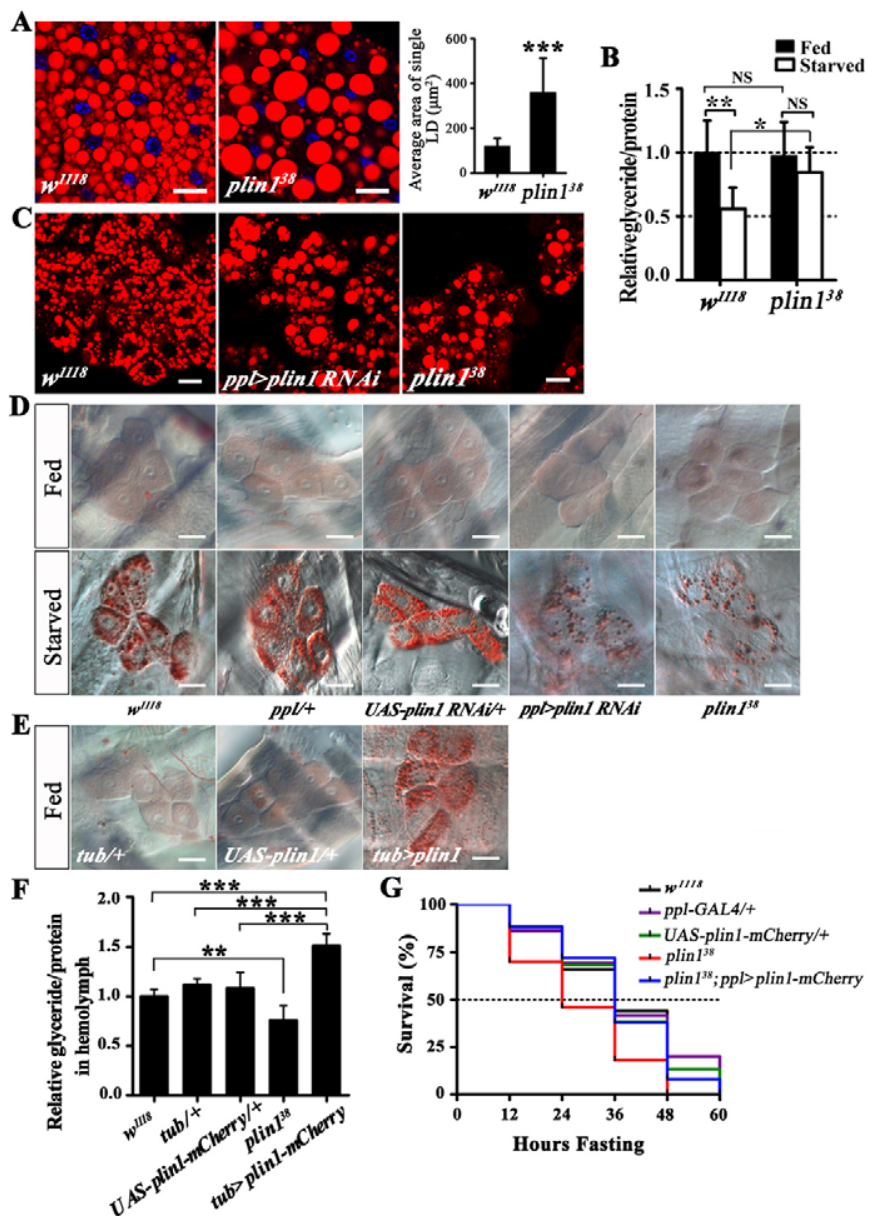

Fig. 2. plin1 facilitates lipid mobilization. (A) Nile Red staining (red) of wandering stage third-instar larval fat bodies. Nuclei are stained with DAPI (blue). plin $1^{38}$ mutants have larger lipid droplets than $w^{1118}$ control. The sizes of lipid droplets in $w^{1118}$ control and $p l i n 1^{38}$ mutants were quantified. $* * * P<0.001$. Scale bars: $20 \mu \mathrm{m}$. (B) Total levels of glyceride in $w^{1118}$ control and $p \operatorname{lin} 1^{38}$ under both fed and starved conditions. NS, non-significant; $* P<0.05 ; * * P<0.01$. (C) Nile Red staining (red) of third-instar larval fat bodies. plin $1^{38}$ mutants and $p p l>$ plin 1 RNAi have larger lipid droplets than controls under starved conditions. Scale bars: $20 \mu \mathrm{m}$. (D) Oil Red O staining of third-instar larval oenocytes. Under fed conditions, the staining signal is weak in $w^{1118}$, ppl-GAL4/+, UAS-plin1 RNAi/+ animals and plin $1^{38}$ mutants. Under starved conditions, the oenocytes of $p \operatorname{lin} 1^{38}$ and $p p l>p l i n 1$ RNAi animals have fewer Oil-Red-O-positive lipid droplets than controls. (E) Under fed conditions, the staining signal is weak in tub-GAL4/+ and UAS-plin 1/+ controls. However, strong staining is observed when plin1 is overexpressed with tub-Gal4. Scale bars: $50 \mu \mathrm{m}$ (D,E). (F) Total relative glyceride levels of circulating hemolymph in $w^{1118}$, tub-GAL4/+, UAS-plin1/+, plin1 $1^{38}$ mutants and plin1-mCherry-overexpressing third-instar larvae under fed conditions. $* P<0.05$; *** $P<0.001$. (G) Life span of $w^{1118}$, ppl-GAL4/+, UAS-plin1/+, plin $1^{38}$ and $p p l>$ plin $1-m$ Cherry rescued $p l i n 1^{38}$ mutant larvae under starvation conditions. plin $1^{38}$ mutants are sensitive to starvation $(P<0.001)$. $p p l>p l i n 1-m C h e r r y$ rescued $p l i n 1^{38}$ mutant larvae have normal life-span under starvation compared with controls $(P>0.05)$.

We next addressed whether expressing plin1 under fed conditions is sufficient to promote lipid mobilization. We found that the overexpression of plin1 resulted in strong Oil Red $\mathrm{O}$ staining in oenocytes (Fig. 2E), suggesting that overexpression of plin1 leads to increased lipid mobilization. We further measured the circulating hemolymph lipid levels in 
controls, plin $1^{38}$ mutant and plinl overexpression larvae under fed conditions. Compared to controls, the circulating lipid levels are significantly reduced in $\operatorname{plin} 1^{38}$ mutants (Fig. 2F). In addition, we found that the circulating lipid levels were dramatically increased in plin1 overexpression larvae, consistent with the strong Oil Red O staining in oenocytes (Fig. 2F). Taken together, these results support that PLIN1 facilitates lipid mobilization.

What is the consequence of defective lipid mobilization in plin1 mutants? Since fats released from lipid droplets are important for animal survival during starvation, we examined the life span of $p \operatorname{lin} 1^{38}$ mutant larvae under starved conditions. We found that the life-span of $p \operatorname{lin} 1^{38}$ mutants was significantly shorter than that of the wild type (Fig. 2G). Since the total glyceride levels are not lower in plin $1^{38}$ mutant larvae compared to the wild type (Fig. 2B), the short life span of $p$ lin $1^{38}$ mutant larvae during starvation is unlikely due to reduced glyceride levels. Therefore, defective lipid mobilization in plin 1 mutants likely makes larvae sensitive to starvation. In addition, the short life span phenotype of $\operatorname{plin} 1^{38}$ mutant larvae can be rescued by fat body-specific expression of PLIN1-mCherry, supporting that the starvation sensitivity of $\operatorname{plin} 1^{38}$ mutants is caused by reduced lipid mobilization in the fat body (Fig. 2G).

\section{dHSL is important for lipid mobilization}

To investigate the possible mechanisms of plin1 in facilitating lipid mobilization, we turned to lipases, which play essential roles in lipolysis. BMM is the Drosophila homolog of ATGL. $\mathrm{bmm}$ mutants have reduced lipolysis and become progressively obese. Moreover, the $\mathrm{bmm}$ mutation enhances the adult obese phenotype of plin1 mutants, indicating that the facilitation of lipolysis by plin 1 is not fully dependent on $\mathrm{bmm}$ (Beller et al., 2010). Indeed, in plin1 mutants, BMM is enriched on lipid droplets (Beller et al., 2010). These findings cannot fully explain the large lipid droplet and reduced fat mobilization phenotype of plin1 mutants. Hence, we focused on HSL.

CG11055/dHSL is the sole homolog of HSL in Drosophila (Kühnlein, 2011). The amino acid sequence of dHSL shares $39 \%$ identity and $56 \%$ similarity to human HSL by BLAST comparison (supplementary material Fig. S4). The active sites in the C-terminal catalytic domain of mammalian HSL are conserved in dHSL (Ser-473, Asp-794 and His-824). However, the five phosphorylation sites found in mouse and human HSL are not conserved in dHSL (supplementary material Fig. S4). We first checked the expression profile of $d H S L$. Northern blot analysis reveals that $d H S L$ is expressed at all developmental stages (Fig. 3A). RNA in situ hybridization in embryos shows the ubiquitous distribution of $d H S L$ transcript at blastoderm stage, which suggests a maternal contribution of the gene. At midembyronic stages, dHSL transcript is detected at parts of the embryonic digestive tract (Fig. 3B). Quantitative RT-PCR analysis shows that $d H S L$ is widely expressed in different tissues of third instar larvae (Fig. 3C). Since mammalian HSL is important for stimulated lipolysis, we then compared $d H S L$ transcription levels in third-instar larvae under both fed and starved conditions. Similar to $\mathrm{bmm}$, the transcription level of $d H S L$ is significantly up-regulated upon starvation (Fig. 3D).

We isolated a $d H S L$ mutant by transposon imprecise excision from transposon line EY20067. $\mathrm{dHSL}^{b 24}$ is a 472 bp deletion which includes the start codon ATG (Fig. 3E). The transcription level of $d H S L$ is greatly reduced in $d H S L^{b 24}$ mutants, suggesting that it is a strong loss-of-function or null allele of $d H S L$ (Fig. 3F;
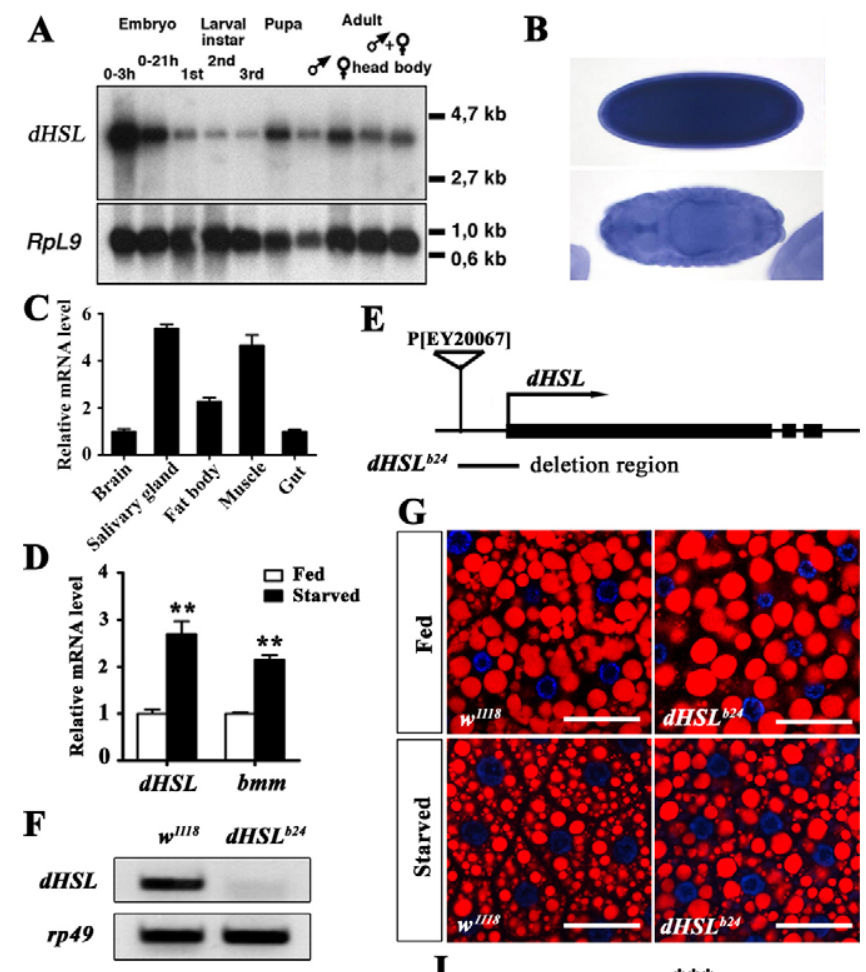

G

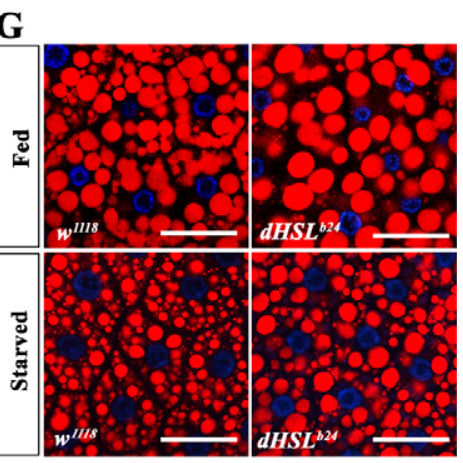

H.

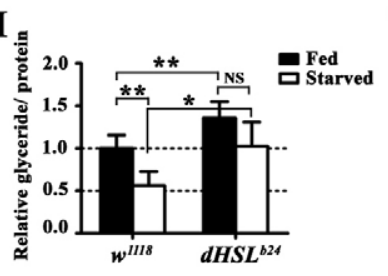

I

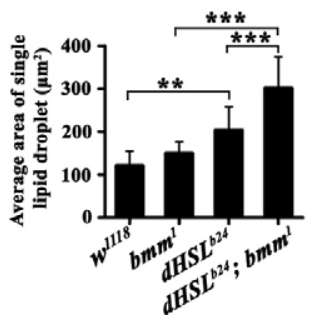

Fig. 3. dHSL is a key lipase in fat mobilization. (A) Northern blot analysis reveals a single $d H S L$ transcript with an estimated size of $4 \mathrm{~kb}$ present at all developmental stages. The strong enrichment during early embryogenesis suggests a maternal contribution of $d H S L$ mRNA. RpL 9 expression was used as normalization control. (B) Ubiquitous distribution of $d H S L$ transcript in blastoderm stage embryos detected by RNA in situ hybridization analysis. In mid-embryonic stages, $d H S L$ mRNA is found in parts of the embryonic digestive tract. (C) Relative mRNA level of $d H S L$ in different tissues of third instar larvae assayed by quantitative RT-PCR. (D) Relative mRNA level of $d H S L$ and $\mathrm{bmm}$ in third-instar larvae under both fed and starved conditions assayed by quantitative RT-PCR. ${ }^{* *} P<0.01$. (E) The genomic structure of the $d H S L$ locus. Black box, coding region. The positions of the transposon insertion EY20067 and the 472 bp deletion of $d H S L^{b 24}$ are indicated. (F) RTPCR of $d H S L$. $d H S L^{b 24}$ is a strong loss of function or null allele. (G) Nile Red staining (red) of third-instar larval fat bodies. Nuclei are stained with DAPI (blue). dHSL ${ }^{b 24}$ mutants have larger lipid droplets than $w^{1118}$ controls under both fed and starved conditions. Scale bar: $50 \mu \mathrm{m}$. (H) Relative glyceride levels of wild-type and $d H S L^{b 24}$ third-instar larvae under both fed and starved conditions. NS, non-significant; $* P<0.05$; $* * P<0.01$. (I) Quantification of lipid droplet size in $\mathrm{bmm}^{1}, d H S L^{b 24}$ and $\mathrm{bmm}^{1} ; d H S L^{b 24}$ backgrounds. Animals with mutations of two lipolytic genes, $b m m$ and $d H S L$, have larger lipid droplets than either single mutant. ${ }^{* *} P<0.01 ; * * * P<0.001$.

supplementary material Fig. S2). $d H S L^{b 24}$ mutant larvae have slightly larger lipid droplets than the wild type under fed conditions (Fig. 3G,I). Moreover, under starved conditions, $d H S L^{b 24}$ mutant larvae contain significantly larger lipid droplets than the wild type (Fig. 3G), suggesting that $d H S L$ 
mutants have lipolysis defects. To quantitatively analyze the lipid mobilization defects in $d H S L$ mutants, the total glyceride level in $d H S L^{b 24}$ mutant larvae was compared to that in the wild type under both fed and starved conditions. $d H S L^{b 24}$ mutants have $30 \%$ more total glyceride than the wild type under fed conditions, and under starved conditions the level of total glyceride of $d H S L^{b 24}$ mutants is doubled compared to the wild type (Fig. $3 \mathrm{H}$ ). Altogether, these results demonstrate that lipid mobilization is impaired in $d H S L$ mutants.

To visualize the dynamics of dHSL in vivo, we created a dHSL-EGFP transgene. Since the lipid droplet phenotype of $d H S L^{b 24}$ mutants can be fully rescued by the functional dHSLEGFP transgene (supplementary material Fig. S2), the localization patterns of dHSL-EGFP in larval fat bodies were examined under different conditions in the wild type or $d H S L^{b 24}$ mutant background. Under fed conditions, dHSL-EGFP signals are largely dispersed in the cytosol with a few puncta on or near the lipid droplets, consistent with the role of dHSL in stimulated lipolysis (Fig. 4A; supplementary material Fig. S5). Under starved conditions, more dHSL-EGFP signal is found on the lipid droplet surface, and appears as rings surrounding the lipid droplets (Fig. 4A; supplementary material Fig. S5). This starved induced lipid droplet localization is consistent with the known function of mammalian HSL in stimulated lipolysis (Egan et al.,
1992), suggesting that the basic regulation of stimulated lipolysis is likely conserved from Drosophila to mammals. In addition, the large lipid droplet phenotype of $d H S L^{b 24}$ mutants can be further enhanced by mutation of $\mathrm{bmm}$ (Fig. 3I), suggesting that mutation of $d H S L$ or $\mathrm{bmm}$ alone only partially impairs lipolysis. Taking these results together, we conclude that dHSL is a key player in lipid mobilization in Drosophila.

\section{PLIN1 is important for localization of dHSL to the surface of lipid droplets}

With the help of the dHSL-EGFP reporter and $d H S L^{b 24}$ mutants, we were able to explore the connection between plin1 and $d H S L$. Since under fed conditions little dHSL-EGFP is localized on the surface of lipid droplets, we were unable to detect any difference in dHSL-EGFP distribution between the wild type and plin $1^{38}$ mutants (Fig. 4A-C; supplementary material Fig. S5). However, under starved conditions, the lipid droplet surface localization of dHSL-EGFP, in particular in large lipid droplets, is absent in plin $1^{38}$ mutants, (Fig. 4A-C; supplementary material Fig. S5). In contrast, under fed conditions, dHSL-EGFP signal can already be found on the surface of some lipid droplets in plin $2^{\text {KG00149 }}$ mutants (supplementary material Fig. S5). In addition, the localization of dHSL-EGFP to the lipid droplet surface under starvation is normal in plin2 $2^{K G 00149}$ mutants (supplementary
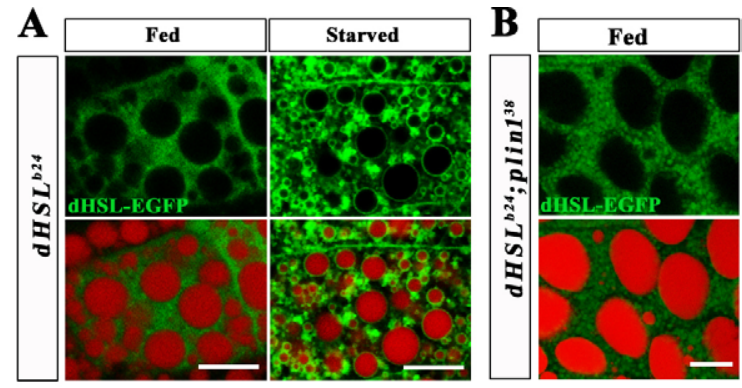

D

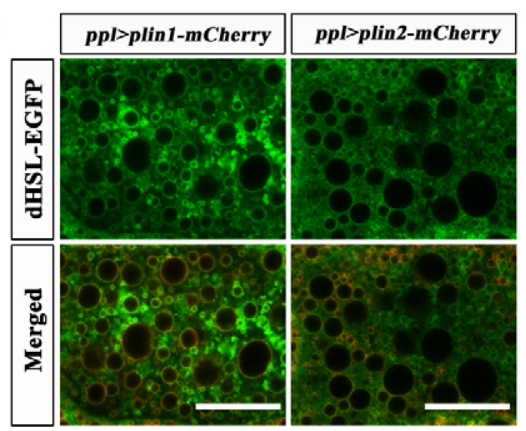

$\mathbf{F}$

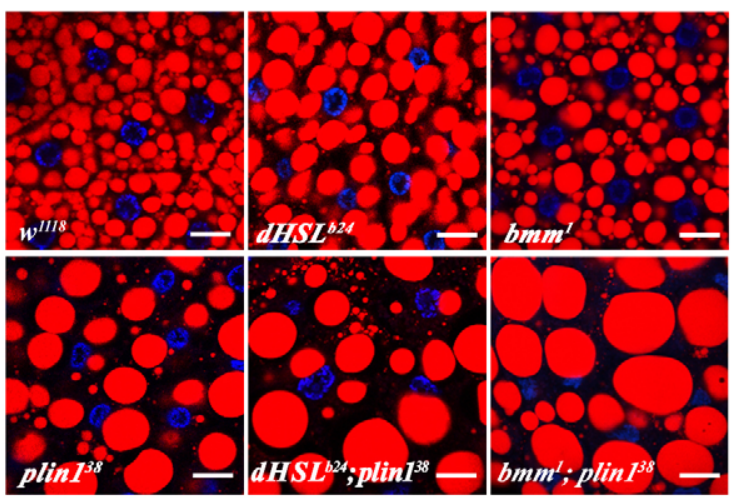

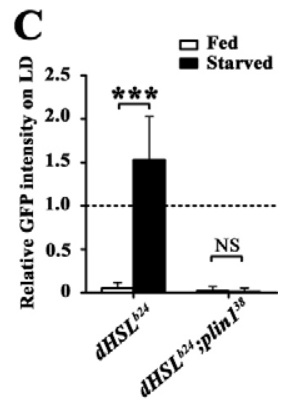

$\mathbf{E}$
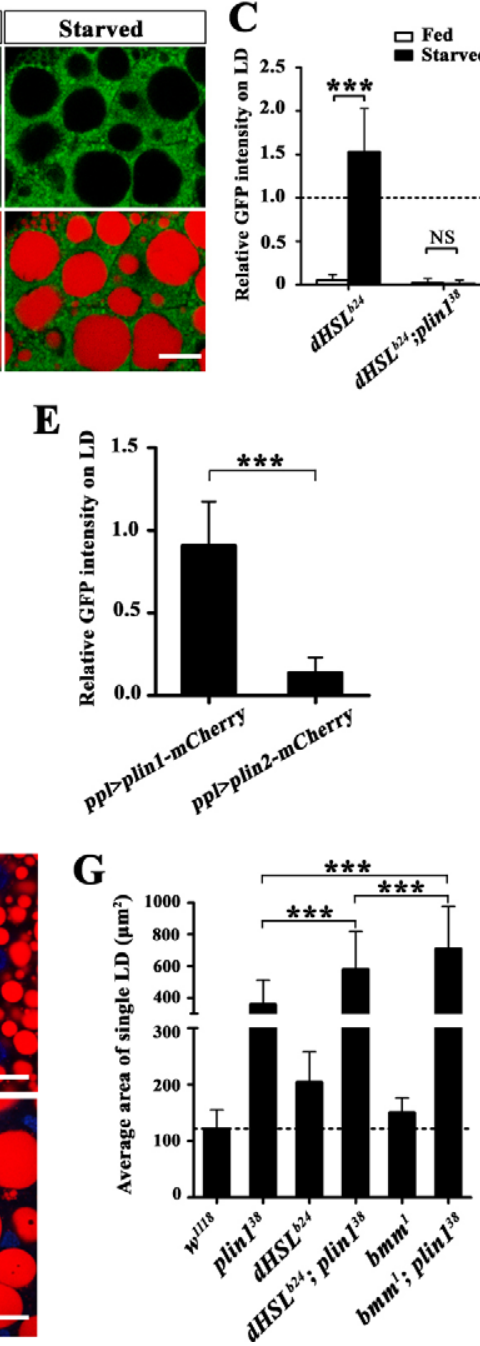

Fig. 4. PLIN1 is required for lipid droplet localization of dHSL. $(A, B)$ Localization of dHSL-EGFP in $d H S L^{b 24}$ and $d H S L^{b 24} ; \operatorname{plin}^{38}$ mutant third-instar larval fat bodies under both fed and starved conditions. LipidTOX is a fluorescent dye that stains neutral lipids. Scale bar: $20 \mu \mathrm{m}$. (C) Quantification of the lipid droplet localization of dHSL-EGFP. The lipid droplet surface localization of dHSL-EGFP under starved conditions is absent in $d H S L^{b 24} ;$ plin $^{38}$ double mutants. NS, nonsignificant; $* * * P<0.001$. (D) plin 1 overexpression leads to more dHSL-EGFP localized to lipid droplets compared with the plin2 overexpression control. Scale bar: $20 \mu \mathrm{m}$. (E) Quantification of the lipid droplet localization of dHSL-EGFP. ${ }^{* * *} P<0.001$. (F) Nile Red staining (red) of wandering stage third-instar larval fat bodies in different genetic backgrounds. Nuclei are stained with DAPI (blue). Scale bar: $20 \mu \mathrm{m}$. (G) Quantification of the size of lipid droplets in different genetic backgrounds. Both $d H S L$ and $b m m$ mutations enhance the large lipid droplet phenotype of plin $1^{38}$ mutants. $* * * P<0.001$. 
material Fig. S5). Therefore, it is possible that PLIN1 facilitates the lipid droplet localization of dHSL-EGFP under starvation and PLIN2 prevents the access of dHSL-EGFP to lipid droplets under fed condition. We further tested this hypothesis by overexpression studies. We found that plin1 overexpression leads to more dHSL-EGFP localized to lipid droplets under fed conditions compared to plin2 overexpression control (Fig. 4D,E). A previous study in mice showed that Perilipin1 is essential for HSL translocation (Sztalryd et al., 2003). Our results here indicate that the regulation of HSL-mediated stimulated lipolysis by perilipins is likely conserved from Drosophila to mammals.

Since plin 1 mutants have larger lipid droplets than $d H S L$ single mutants, the function of PLIN1 cannot be fully dependent on dHSL. When we made $p \operatorname{lin} 1^{38} ; d H S L^{b 24}$ double mutants, we found that the large lipid droplet phenotype in $p \operatorname{lin} 1^{38}$ mutant larvae was slightly enhanced (Fig. 4F,G). Consistent with a previous report (Beller et al., 2010), $\mathrm{bmm}^{1}$ mutants can also enhance the $\operatorname{plin} 1^{38}$ mutant phenotype (Fig. 4F,G). These results indicate that under normal conditions, dHSL and BMM functions may not be fully dependent on PLIN1. In addition, considering that $\operatorname{plin} 1^{38}$ mutants have larger lipid droplets than $\mathrm{bmm}^{1}$; $\mathrm{dHSL}^{\mathrm{b24}}$ double mutants (Fig. 2A, Fig. 3I), it is possible that plin 1 has functions independent of both $d H S L$ and $b m m$. This conclusion is consistent with previous finding that PLIN1 may have a structural role in lipid droplets (Beller et al., 2010). Alternatively, PLIN1 may affect localizations of lipase other than dHSL and BMM.

\section{Redundant functions of PLIN1 and PLIN2}

The different localization patterns and opposing functions of PLIN1 and PLIN2 lead to a model in which PLIN2 protects small lipid droplets from BMM-mediated lipolysis in wild-type larvae, while PLIN1 promotes dHSL-mediated lipolysis in large lipid droplets (Fig. 5A, wild-type model). To test this hypothesis, we overexpressed plin 2 in the plin1 mutant background and vice versa. Our model predicts that plin2 overexpression could potentially enhance the plin1 mutant large lipid droplet phenotype by protecting lipid droplets from lipolysis. On the other hand, plin 1 overexpression could enhance the plin 2 mutant small lipid droplet phenotype by promoting lipid mobilization. Indeed, we found that overexpression of plin2 in the plin $1^{38}$ mutant background resulted in even larger lipid droplets in the larval fat body than those in flies homozygous for $\operatorname{plin}^{38}$ alone (Fig. 5B). Surprisingly, although overexpression of plin1 alone results in small lipid droplets (supplementary material Fig. S1), it did not enhance, but instead mildly suppressed, the small lipid droplet phenotype in plin2 $2^{K G 00149}$ mutant larvae (Fig. 5C). These results suggest that in the plin2 mutant background, PLIN1 may have the same function as PLIN2 (Fig. 5A, plin2 mutant model).

We then made plin1;plin2 double mutants to further examine this possibility. If plin 1 and plin2 have opposite functions and act in separate pathways, we might expect that the phenotype of plin1;plin2 double mutants would be intermediate to either single mutant. If plin1 and plin2 have opposite functions in the same linear pathway, the phenotype of plin1;plin2 double mutants should resemble either the plin1 or the plin2 single mutant. Lastly, if plin1 and plin2 have redundant functions as we hypothesized (Fig. 5A, plin2 mutant model), the phenotype of plin1;plin2 double mutants should be stronger than the plin2 single mutant. We found that $p \operatorname{lin} 1^{38} ;$ plin2 $2^{K G 00149}$ double mutant larvae display an even smaller lipid droplet phenotype than the
A

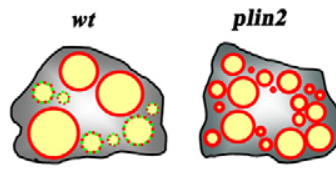

PLIN1 PLIN2 PLIN1 DK/2

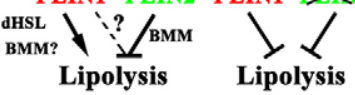

B
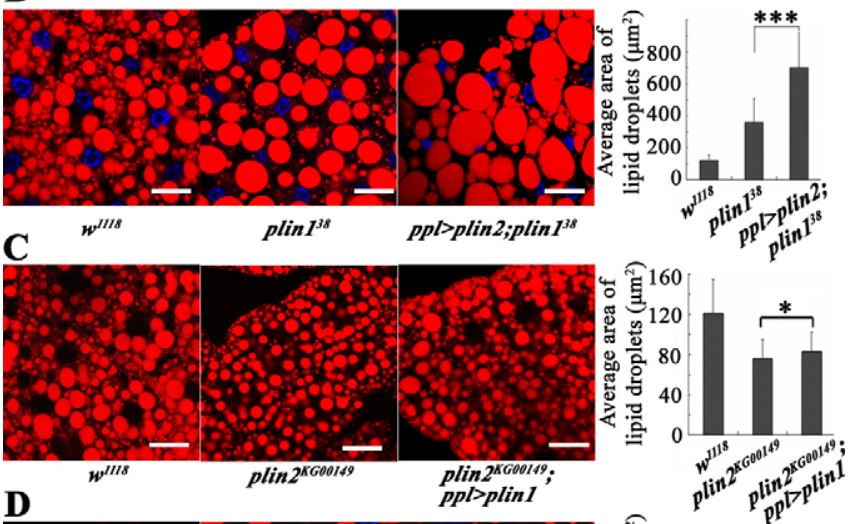

D

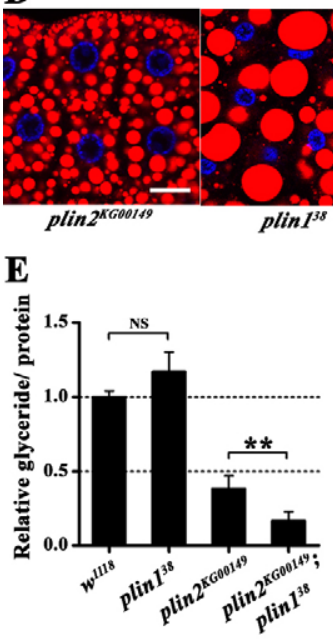

ppl>plin1

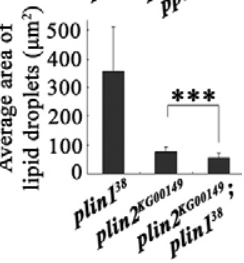

F Fed

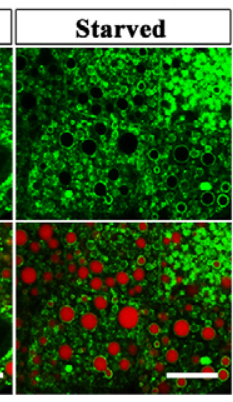

Fig. 5. PLIN1 has both redundant and opposite roles to PLIN2.

(A) Models of the functions of PLIN1 and PLIN2 in lipid droplet homeostasis In the wild type, PLIN1 mainly promotes lipolysis of PLIN2-negative large lipid droplets through dHSL. In small lipid droplets, PLIN2 might prevent BMM-mediated lipolysis. In plin2 mutants, the lipid droplets are small and PLIN1 might prevent lipolysis. (B-D) Nile Red staining (red) of wandering stage third-instar larval fat bodies. Nuclei are stained with DAPI (blue). Scale bars: $20 \mu \mathrm{m}$. (B) Overexpression of plin2 enhances the plin1 mutant large lipid droplet phenotype. $* * * P<0.001$. (C) Overexpression of plin1 slightly suppresses the plin2 mutant small lipid droplet phenotype. $* P<0.05$

(D) plin 1;plin2 double mutants show smaller lipid droplets than plin 2 single mutants. $* * * P<0.001$. (E) Relative glyceride levels in different genetic backgrounds. plin1;plin2 double mutants have lower glyceride level than plin2 single mutants. NS, non-significant; $* * P<0.01$. (F) Lipid droplet localization of dHSL-EGFP in $p \operatorname{lin} 1^{38}$ and plin $2^{K G 00149}$ double mutant thirdinstar larval fat bodies under both fed and starved conditions. Scale bars: $20 \mu \mathrm{m}$

plin $2^{K G 00149}$ single mutant, suggesting a redundant function of PLIN1 and PLIN2 (Fig. 5D). To further confirm this finding, we also measured the total glyceride levels of $p \operatorname{lin} 1^{38} ;$ plin $2^{\text {KG00149 }}$ double mutant larvae. Consistent with the lipid droplet staining 
results, the total glyceride levels of $p \operatorname{lin} 1^{38} ;$ plin2 $2^{K G 00149}$ double mutant larvae are significantly lower than those of plin2 $2^{K G 00149}$ single mutants (Fig. 5E). Moreover, in fat body cells of plin $1^{38}$ and plin $2^{K G 00149}$ double mutant larvae, even under fed condition, dHSL-EGFP can localize to lipid droplets surface (Fig. 5F; supplementary material Fig. S5). The enhancement of the plin2 mutant phenotype by mutation of plin1 suggests that PLIN1 has an anti-lipolytic function that is partially redundant with PLIN2 and this function is revealed when plin2 function is absent (Fig. 5A, plin2 mutant model).

\section{The C-terminal region of PLIN1 confers its functional specificity}

Since PLIN1 and PLIN2 belong to the same protein family, what make these two proteins so different in function? Compared to PLIN2, PLIN1 has an extended C-terminal region (Fig. 6A). We analyzed the function of this $\mathrm{C}$-terminal region with domain deletion and swapping experiments. The protein localization pattern and the rescuing activity of fluorescent protein-tagged PLIN1, PLIN2 and various chimeric proteins were compared in the plin $1^{38}$ mutant background (Fig. 6A).

We first compared the protein localization patterns of PLIN1 without the C-terminal region (PLIN1 $\Delta C$ ) and PLIN2 fused with the C-terminal region of PLIN1 (PLIN2-PLIN1C). Similar to PLIN1-mCherry, PLIN2-PLIN1C-mCherry is localized to most if not all lipid droplets (Fig. 6B). Additionally, like PLIN2-EYFP, PLIN1 $\triangle \mathrm{C}$-EYFP is mainly localized to small lipid droplets (Fig. 6B). Therefore, the C-terminal region determines the localization pattern of PLIN1 protein.

For unknown reasons, ppl-Gal4 sometimes generates a mosaic pattern of gene expression. Therefore, in the rescue experiments, we were able to compare the size of lipid droplets in PLIN1mCherry-expressing cells and non-expressing cells. We found that cells with mCherry signal had small lipid droplets, while cells without mCherry signal contained large lipid droplets similar to plin $1^{38}$ mutants (Fig. 6C,D). This finding demonstrates that the function of PLIN1 is cell-autonomous. In addition, expressing PLIN2-EYFP does not rescue, but instead further enhances, the plin1 mutant large lipid droplet phenotype (Fig. 6C), consistent with the results from expressing untagged PLIN2 (Fig. 5B). More importantly, the PLIN2-PLIN1C-mCherry transgene, but not the PLIN1 1 C-EYFP transgene, rescues the large lipid droplet phenotype and dHSL-EGFP location defect in $\operatorname{plin} 1^{38}$ mutant larvae (Fig. 6C-E). Together, these results indicate that the $\mathrm{C}$ terminal region of PLIN1 determines the localization and functional difference between PLIN1 and PLIN2.

\section{Discussion}

Although perilipins are important for lipid droplet homeostasis, their exact in vivo roles are not fully clear. Using a genetic approach, we analyzed the functions of the two Drosophila perilipins, PLN1 and PLN2. We found that PLIN1 exhibits opposite and redundant functions with PLIN2. In wild-type larvae, PLIN1 mainly facilitates fat mobilization by regulating the lipid droplet surface localization of dHSL, the Drosophila homolog of mammalian hormonesensitive lipase. In the absence of plin2, plin1 shows plin2-like function in preventing lipid mobilization (Fig. 5A).

The analysis of $d H S L$ reveals several interesting points. Under fed conditions, both the TAG level and the size of lipid droplets are slightly increased in $d H S L$ mutant larvae, indicating that dHSL may function under basal condition. In supporting that,

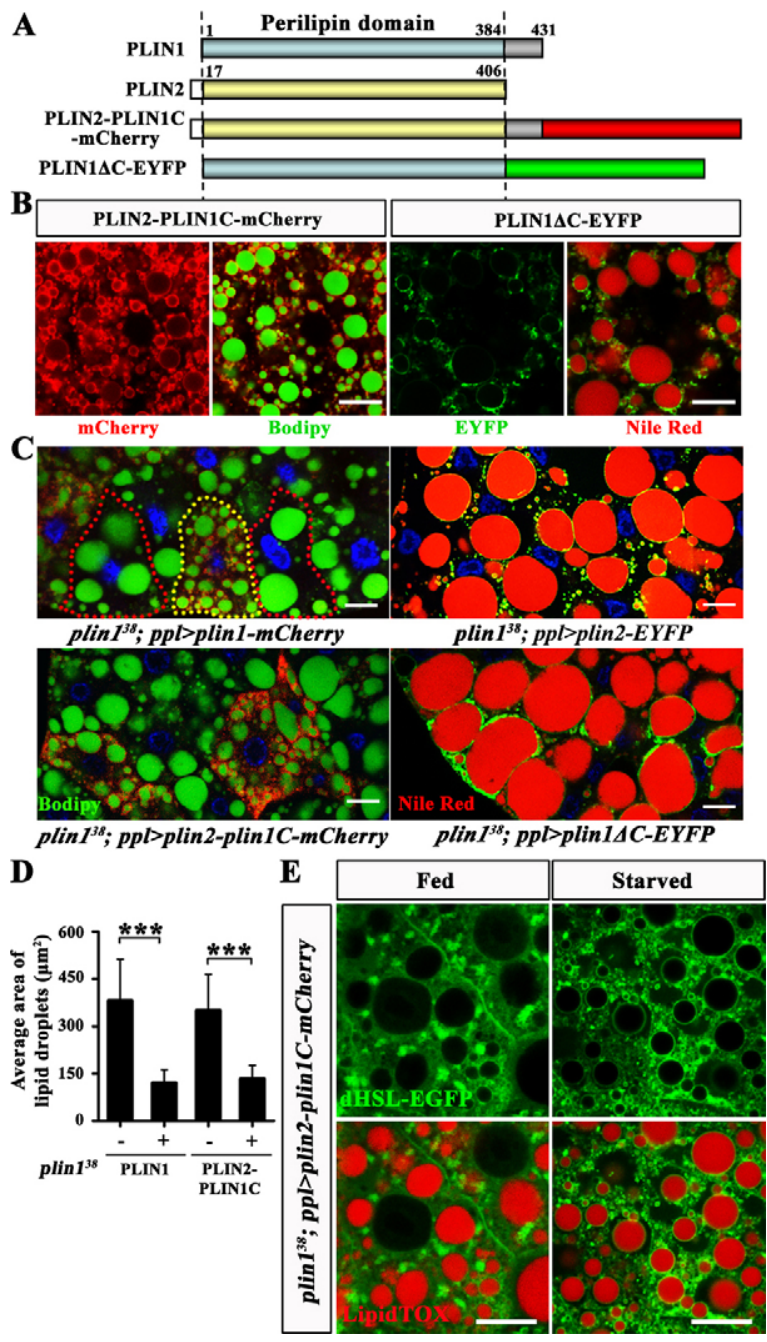

Fig. 6. The PLIN1 C-terminal region determines the functional specificity of PLIN1. (A) Domain structures of PLIN1, PLIN2 and chimeric proteins. The perilipin domain is predicted by the PFAM program.

(B) Fluorescent images of wandering stage third-instar larval fat bodies. The fluorescent proteins are expressed with ppl-Gal4. PLIN2-PLIN1C-mCherry is localized to most if not all lipid droplets (stained green by Bodipy).

PLIN1 1 C-EYFP is mainly localized to small lipid droplets (stained by Nile Red). Scale bars: $20 \mu \mathrm{m}$. (C) Bodipy or Nile Red staining of wandering stage third-instar larval fat bodies. Nuclei are stained with DAPI (blue). The fluorescent proteins are expressed with ppl-Gal4. The dashed yellow line encloses a PLIN1-mCherry-expressing cell; dashed red lines enclose PLIN1mCherry-non-expressing cells. The large lipid droplet phenotype is rescued cell-autonomously by expression of PLIN1-mCherry. Expression of PLIN2PLIN1C-mCherry rescues the large lipid droplet (stained green by Bodipy) phenotype in $\operatorname{plin} 1^{38}$ mutants. PLIN1 $\Delta$ C-EYFP fails to rescue the large lipid droplet (stained by Nile Red) phenotype in plin $1^{38}$ mutants. Scale bar: $20 \mu \mathrm{m}$. (D) Quantification of lipid droplet size in C. $* * * P<0.001$.

(E) Localization of dHSL-EGFP in $p p l>$ plin2-plin $1 C$-mCherry-rescued plin $1^{38}$ mutant third-instar larval fat bodies under both fed and starved conditions. Scale bar: $20 \mu \mathrm{m}$.

dHSL mutation enhances the large lipid droplet phenotype of bmm mutants. The location of dHSL-EGFP to lipid droplets under starvation highlights that the mechanism by which HSL regulates stimulated lipolysis is likely conserved from Drosophila to mammals. We took advantage of the dHSL-EGFP reporter we generated to establish a strong connection between 
defective fat mobilization in plin1 mutants and the lipid droplet surface localization of dHSL.

The fact that plin1 mutant larvae have larger lipid droplets than bmm;dHSL double mutants can be explained by the proposed structural role of PLIN1 in lipid droplets (Beller et al., 2010). Since plin1 mutants have giant lipid droplets, it is possible that PLIN1 may be involved in lipid droplet fission or fusion. Several recent studies have revealed that phosphatidic acid (PA) is important for the formation of supersized lipid droplets in Seipin mutants (Fei et al., 2011; Tian et al., 2011). It remains to be determined whether PLIN1 affects the metabolism of fatty acids or phospholipids, such as PA. Moreover, our results also extend previous findings (Beller et al., 2010) by showing that PLIN1 has PLIN2-like function in protecting lipid droplets from lipolysis (Fig. 5A). Currently, we do not know how PLIN1 performs this protective role. It is possible that it acts by blocking the access of BMM. Previous finding that more BMM localizes to lipid droplets in plin1 mutants is consistent with this possibility (Beller et al., 2010).

The dual role of Drosophila PLIN1 prompts comparison between Drosophila PLIN1 and mammalian Perilipin1. Both PLIN1 and Perilipin1 have two opposing functions in lipid droplets: preventing lipolysis and facilitating lipolysis. The two roles of Perilipin1 are regulated by phosphorylation. Unphosphorylated Perilipin 1 protects lipid droplets from lipolysis by blocking the access of lipases, while phosphorylated Perilipin1 releases the ATGL activator CGI58, resulting in activation of ATGL, which promotes lipolysis (Zimmermann et al., 2004). Phosphorylated Perilipin1 can also elicit translocation of HSL from the cytosol to the lipid droplet surface (Sztalryd et al., 2003). Similarly, studies using purified Drosophila PLIN1 implied that PKA phosphorylation of PLIN1 had a direct effect on lipase activity (Arrese et al., 2008; Patel et al., 2005). Moreover, we found that PLIN1 is important for dHSL lipid droplet location. Therefore, the regulation of HSL localization by perilipins is likely highly conserved from Drosophila to mammals. It remains to be determined whether PLIN1 regulates the activity of BMM, the Drosophila ATGL. On the other hand, Drosophila plin1 differs from mouse Plin1 in the following ways. First, unlike Plin1knockout mice (Martinez-Botas et al., 2000), Drosophila plin1 mutants are not lean; indeed, a recent study showed that plin1 mutant animals develop adult-onset obesity (Beller et al., 2010). Second, overexpression of Plin1 results in aggregated lipid droplets (Marcinkiewicz et al., 2006), whereas overexpression of plinl leads to small lipid droplets. Lastly, the partially redundant function of PLIN1 was revealed in the plin2 mutant background. It is not known whether Perilipin1 has other functions in the absence of other perilipins in vivo.

Our results hypothesize that PLIN2, together with PLIN1, may protect small lipid droplets at an early stage of lipid droplet biogenesis from BMM- and probably dHSL-mediated lipolysis, while PLIN1 facilitates dHSL-mediated lipolysis in large lipid droplets. Based on our phenotypic analysis, it is conceivable that the major function of PLIN1 is in facilitating fat mobilization. Because large lipid droplets have greater lipid content, lipolysis of large droplets may be an efficient way to support the cell's energy needs and to balance lipid usage with lipid droplet biogenesis. Such fine regulation is important for maintaining lipid homeostasis. Moreover, the functional complexity of PLIN1 may reflect the evolution of ancient perilipins from simple barriers that protect lipid droplets to more active regulators of lipid homeostasis.
How are the dual functions of PLIN1 regulated? It is possible that PLIN1 may have different structures/states and binding partner(s) in lipid droplets of different sizes. Phosphorylated PLIN1 was found to affect the activity of lipase in in vitro assays (Arrese et al., 2008). Therefore, the phosphorylation state of PLIN1 may be different in small and large lipid droplets. Although the functional importance of PLIN1 phosphorylation remains to be determined in vivo, a recent study showed that the canonical PKA target sites are not important for PLIN1 function (Beller et al., 2010). Therefore, identification of the phosphorylation site of PLIN1 will lead to better understanding of the regulation of PLIN1 function. Since the C-terminal region of PLIN1 determines its functional specificity, regulation of the dual role may be a property of the $\mathrm{C}$ terminus. The N-terminal portion of PLIN1 may be sufficient for its function in protecting lipid droplets from lipolysis. The C-terminal region of PLIN1 is highly conserved among Drosophila species (supplementary material Fig. S6). Identifying protein partners of the C-terminal region could help to reveal the regulatory mechanisms involved. Similarly, compared to ADRP and TIP47, Perilipin1 has an extended C-terminal region. Phosphorylation of key residues in the $\mathrm{C}$-terminal region of Perilipin1 is important for ATGL activation and lipid droplet dispersal (Marcinkiewicz et al., 2006; Miyoshi et al., 2007). Frame-shift mutations at the C-terminal region of Perilipin1 result in dominant partial lipodystrophy in human, supporting the functional importance of the C-terminal region (Gandotra et al., 2011).

Our study reveals the functions of the only two perilipins in Drosophila. The fact that plin1;plin2 double mutants have small lipid droplets indicates that perilipins are dispensable for the initial biogenesis of lipid droplets, but are required for the growth of lipid droplets. Together with a recent study on PLIN1 (Beller et al., 2010), these findings provide a better understanding of the exact function of perilipins in vivo. plin1, plin2 and $d H S L$ mutants can be used as models to further probe the homeostasis of lipid droplets. More functional studies of Drosophila lipidrelated genes may facilitate a deeper understanding of diseases related to fat metabolism, such as obesity and diabetes.

\section{Materials and Methods}

\section{Drosophila stocks and husbandry}

All flies were propagated at $25^{\circ} \mathrm{C}$ on standard cornmeal food with Angel dry yeast (Angel Yeast, Hubei, China). All the plinl alleles were generated from the transposon insertion line MB10034 through Minos transposon imprecise excision. These alleles were identified by RT-PCR. $d H S L^{b 24}$ was generated by transposon imprecise excision from the line $d H S L^{E Y 20067}$. The molecular lesions of mutants were determined by PCR and sequencing. Unless specified, Drosophila stocks were obtained from the Bloomington Stock Center and the Vienna Drosophila RNAi Center. $w^{1118}$ was used as the wild-type control.

\section{Molecular biology}

For $p U A S T-d H S L-E G F P$, the $d H S L$ coding region (without the stop codon) was fused with EGFP and then $d H S L-E G F P$ was inserted into $p U A S T$ through $B g l \mathrm{II}$ and $X b a \mathrm{I}$ sites. The domain swapping construct pUAST-attB-plin2-plin 1C$m$ Cherry was generated from pUAST-attB-plin1-mCherry. First, the plin1 sequence was replaced by a plin2 cDNA without the stop codon through EcoRI and KpnI sites to generate $p U A S T$-attB-plin2-mCherry. The C-terminus of plin1 (encoding aa 385-431) was then inserted into the KpnI site. pUAST-attB-plin2$E Y F P$ and $p U A S T$-attB-plin $1 \triangle C$-EYFP were generated by ligating the plin 2 coding region or plin1 $\triangle C$ (encoding aa 1-384) in frame into an EYFP vector through EcoRI and KpnI sites. The plin2-EYFP or plin1 $\triangle C$-EYFP fragment was shuttled into the transformation vector $p U A S T$-attB through EcoRI and $X b a \mathrm{I}$ sites. All constructs requiring PCR amplification were confirmed by sequencing. All quantitative RT-PCRs were performed on an ABI PRISM 7900HT real-time cycler (Applied Biosystems) using Power SYBR Green PCR Master Mix (Applied Biosystems). Primer sequences are available upon request.

Developmental Northern blot analysis was essentially done as described (Grönke et al., 2003) using $2 \mu \mathrm{g}$ polyA ${ }^{+}$RNA for each developmental stage. A 
radioactively labeled $d H S L$ antisense RNA probe was prepared by in vitro transcription on a HindIII-linearized RE52776 (Drosophila Genomics Resource Center; corresponding to $d H S L$ isoform RB annotated in FlyBase) $d H S L$ cDNA template by using T3 polymerase and the Strip-EZ RNA kit (Ambion). For normalization, the blot was reprobed with an antisense RNA probe detecting ribosomal protein $R p L 9$ transcripts as described (Grönke et al., 2003).

\section{Whole-mount in situ hybridization}

In situ hybridization on whole-mount wild-type embryos was essentially performed as described (Grönke et al., 2003). A digoxigenin-labeled dHSL antisense RNA probe was prepared by in vitro transcription of NotI-linearized dHSL cDNA RE52776 1-1664 (a 3'-truncated version of RE52776) with T3 polymerase. Stained embryos were dehydrated by an ethanol series, embedded in Canada balsam and imaged using a Zeiss Axiophot microscope equipped with a Kontron ProgRes 3012 camera.

\section{Staining and microscopy}

For lipid droplet staining, larvae were dissected in PBS and fixed in $4 \%$ paraformaldehyde in PBS for $30 \mathrm{~min}$ at room temperature. Tissues were then rinsed twice with $1 \times$ PBS, incubated for $30 \mathrm{~min}$ in either a 1:1000 dilution with PBS of 0.05\% Nile Red (Sigma), $1 \mathrm{mg} / \mathrm{ml}$ BODIPY 493/503 (Invitrogen), 1:200 dilution with PBS of LipidTOX Deep Red (Invitrogen) or Oil Red O staining solution (6 ml of $0.1 \%$ Oil Red O in isopropanol and $4 \mathrm{ml}$ distilled water: prepared fresh and passed through a $0.45 \mu \mathrm{m}$ syringe filter) and then rinsed twice with distilled water. $2 \mathrm{ng} / \mu \mathrm{l}$ DAPI was used to stain nuclei. Stained samples were mounted in $75 \%$ glycerol for microscopy analysis. To quantify lipid droplet size, the area of the three largest lipid droplets per cell from 50 fat body cells was measured by NIS-Elements BR 3.0. To quantify the lipid droplet surface localization of fluorescence proteins (PLIN1mCherry, PLIN2-EYFP and dHSL-EGFP), the fluorescence intensity on the surface of lipid droplets of 30 fat body cells was measured by NIS-Elements BR 3.0 and normalized to the cytosolic intensity.

\section{Glyceride quantification}

Glyceride quantification was performed as previously described previously (Palanker et al., 2009). For total glyceride, eight third-instar larvae (six groups for each genotype) were used. For circulating glyceride, hemolymph was collected from fifteen L3 larvae (six groups for each genotype) and diluted in $50 \mu \mathrm{l}$ PBST $(0.05 \%$ Tween 20$)$, heated at $70^{\circ} \mathrm{C}$ for $15 \mathrm{~min}$, and centrifuged at 12,000 r.p.m. for $5 \mathrm{~min}$. Glyceride in the hemolymph supernatant was measured using TAG determination kits (Sigma).

\section{Starvation test}

Wild-type and mutant embryos were collected within a $4 \mathrm{hr}$ period and raised at low density on standard fly food at $25^{\circ} \mathrm{C} .72 \mathrm{hrs}$ after hatching, larvae were either fed with normal food or starved on paper soaked in PBS for $12 \mathrm{hrs}$. Fed and starved larvae were then dissected and stained with Nile Red or Oil Red O. For the life span test, $72 \mathrm{hrs}$ after hatching, three batches of 60 larvae for each genotype were starved on paper soaked in PBS. The mortality rates were determined by regularly counting the number of dead larvae. 86 90 hrs after hatching larvae were either fed with normal food or starved on paper soaked in PBS for $24 \mathrm{hrs}$ and then collected for total glyceride measurement. For dHSL-EGFP location assay, larvae at $86 \sim 90 \mathrm{hrs}$ after hatching were either fed with normal food or starved on PBS soaked paper for 6 hrs and then dissected and stained with LipidTOX for analysis.

\section{Statistical analyses}

All quantitative data unless otherwise specified are statistically analyzed using Student's $t$-test or ANOVA with a post Tukey's multiple comparison test. All data are reported as the mean \pm s.d. Survival curves for starvation test were generated using GraphPad Prism 5.0 and $P$ values were calculated using the log rank (Mantel-Cox) test.

\section{Acknowledgements}

We thank Pierre Leopold for $p p l$-Gal4 flies. We thank Weijie Liu for the help in generating $d H S L$ mutants.

\section{Funding}

Our research is supported by Ministry of Science and Technology of China [grant number 2009CB919000]; and National Natural Science Foundation of China [grant numbers 31071253 and 30830069]. X.H. is funded by the One Hundred Talent project from the Chinese Academy of Sciences.

\section{Supplementary material available online at}

http://jcs.biologists.org/lookup/suppl/doi:10.1242/jcs.101329/-/DC1

\section{References}

Arrese, E. L., Rivera, L., Hamada, M., Mirza, S., Hartson, S. D., Weintraub, S. and Soulages, J. L. (2008). Function and structure of lipid storage droplet protein 1 studied in lipoprotein complexes. Arch. Biochem. Biophys. 473, 42-47.

Baker, K. D. and Thummel, C. S. (2007). Diabetic larvae and obese flies-emerging studies of metabolism in Drosophila. Cell Metab. 6, 257-266.

Barbieri, M., Bonafe, M., Franceschi, C. and Paolisso, G. (2003). Insulin/IGF-Isignaling pathway: an evolutionarily conserved mechanism of longevity from yeast to humans. Am. J. Physiol. Endocrinol. Metab. 285, E1064-E1071.

Beller, M., Sztalryd, C., Southall, N., Bell, M., Jackle, H., Auld, D. S. and Oliver, B. (2008). COPI complex is a regulator of lipid homeostasis. PLoS Biol. 6, e292.

Beller, M., Bulankina, A. V., Hsiao, H. H., Urlaub, H., Jackle, H. and Kuhnlein, R. P. (2010). PERILIPIN-dependent control of lipid droplet structure and fat storage in Drosophila. Cell Metab. 12, 521-532.

Bickel, P. E., Tansey, J. T. and Welte, M. A. (2009). PAT proteins, an ancient family of lipid droplet proteins that regulate cellular lipid stores. Biochim. Biophys. Acta 1791, 419-440.

Boström, P., Andersson, L., Rutberg, M., Perman, J., Lidberg, U., Johansson, B. R., Fernandez-Rodriguez, J., Ericson, J., Nilsson, T., Boren, J. et al. (2007). SNARE proteins mediate fusion between cytosolic lipid droplets and are implicated in insulin sensitivity. Nat. Cell Biol. 9, 1286-1293.

Bulankina, A. V., Deggerich, A., Wenzel, D., Mutenda, K., Wittmann, J. G., Rudolph, M. G., Burger, K. N. and Honing, S. (2009). TIP47 functions in the biogenesis of lipid droplets. J. Cell Biol. 185, 641-655.

Egan, J. J., Greenberg, A. S., Chang, M. K., Wek, S. A., Moos, M. C., Jr and Londos, C. (1992). Mechanism of hormone-stimulated lipolysis in adipocytes: translocation of hormone-sensitive lipase to the lipid storage droplet. Proc. Natl. Acad. Sci. USA 89, 8537-8541.

Farese, R. V., Jr and Walther, T. C. (2009). Lipid droplets finally get a little R-E-S-PE-C-T. Cell 139, 855-860.

Fauny, J. D., Silber, J. and Zider, A. (2005). Drosophila Lipid Storage Droplet 2 gene (Lsd-2) is expressed and controls lipid storage in wing imaginal discs. Dev. Dyn. 232, $725-732$.

Fei, W., Shui, G., Zhang, Y., Krahmer, N., Ferguson, C., Kapterian, T. S., Lin, R. C., Dawes, I. W., Brown, A. J., Li, P. et al. (2011). A role for phosphatidic acid in the formation of "supersized" lipid droplets. PLoS Genet. 7, e1002201.

Flier, J. S. (2004). Obesity wars: molecular progress confronts an expanding epidemic. Cell 116, 337-350.

Gandotra, S., Le Dour, C., Bottomley, W., Cervera, P., Giral, P., Reznik, Y., Charpentier, G., Auclair, M., Delepine, M., Barroso, I. et al. (2011). Perilipin deficiency and autosomal dominant partial lipodystrophy. N. Engl. J. Med. 364, 740748

Gesta, S., Tseng, Y. H. and Kahn, C. R. (2007). Developmental origin of fat: tracking obesity to its source. Cell 131, 242-256.

Granneman, J. G., Moore, H. P., Krishnamoorthy, R. and Rathod, M. (2009). Perilipin controls lipolysis by regulating the interactions of AB-hydrolase containing 5 (Abhd5) and adipose triglyceride lipase (Atgl). J. Biol. Chem. 284, 34538-34544.

Grönke, S., Beller, M., Fellert, S., Ramakrishnan, H., Jackle, H. and Kuhnlein, R. P. (2003). Control of fat storage by a Drosophila PAT domain protein. Curr. Biol. 13, 603-606

Grönke, S., Mildner, A., Fellert, S., Tennagels, N., Petry, S., Muller, G., Jackle, H. and Kuhnlein, R. P. (2005). Brummer lipase is an evolutionary conserved fat storage regulator in Drosophila. Cell Metab. 1, 323-330.

Guo, Y., Walther, T. C., Rao, M., Stuurman, N., Goshima, G., Terayama, K., Wong, J. S., Vale, R. D., Walter, P. and Farese, R. V. (2008). Functional genomic screen reveals genes involved in lipid-droplet formation and utilization. Nature 453, 657661

Gutierrez, E., Wiggins, D., Fielding, B. and Gould, A. P. (2007). Specialized hepatocyte-like cells regulate Drosophila lipid metabolism. Nature 445, 275-280.

Kimmel, A. R., Brasaemle, D. L., McAndrews-Hill, M., Sztalryd, C. and Londos, C. (2010). Adoption of PERILIPIN as a unifying nomenclature for the mammalian PATfamily of intracellular, lipid storage droplet proteins. J. Lipid Res. 51, 468-471.

Kühnlein, R. P. (2011). The contribution of the Drosophila model to lipid droplet research. Prog. Lipid Res. 50, 348-356.

Marcinkiewicz, A., Gauthier, D., Garcia, A. and Brasaemle, D. L. (2006). The phosphorylation of serine 492 of perilipin a directs lipid droplet fragmentation and dispersion. J. Biol. Chem. 281, 11901-11909.

Martin, S. and Parton, R. G. (2006). Lipid droplets: a unified view of a dynamic organelle. Nat. Rev. Mol. Cell Biol. 7, 373-378.

Martinez-Botas, J., Anderson, J. B., Tessier, D., Lapillonne, A., Chang, B. H., Quast, M. J., Gorenstein, D., Chen, K. H. and Chan, L. (2000). Absence of perilipin results in leanness and reverses obesity in $\operatorname{Lepr}(\mathrm{db} / \mathrm{db})$ mice. Nat. Genet. 26, 474-479.

Miyoshi, H., Souza, S. C., Zhang, H. H., Strissel, K. J., Christoffolete, M. A., Kovsan, J., Rudich, A., Kraemer, F. B., Bianco, A. C., Obin, M. S. et al. (2006). Perilipin promotes hormone-sensitive lipase-mediated adipocyte lipolysis via phosphorylation-dependent and -independent mechanisms. J. Biol. Chem. 281, 15837-15844.

Miyoshi, H., Perfield, J. W., 2nd, Souza, S. C., Shen, W. J., Zhang, H. H., Stancheva, Z. S., Kraemer, F. B., Obin, M. S. and Greenberg, A. S. (2007). Control of adipose triglyceride lipase action by serine 517 of perilipin A globally regulates protein kinase A-stimulated lipolysis in adipocytes. J. Biol. Chem. 282, 996-1002. 
Palanker, L., Tennessen, J. M., Lam, G. and Thummel, C. S. (2009). Drosophila HNF4 regulates lipid mobilization and beta-oxidation. Cell Metab. 9, 228-239.

Patel, R. T., Soulages, J. L., Hariharasundaram, B. and Arrese, E. L. (2005) Activation of the lipid droplet controls the rate of lipolysis of triglycerides in the insect fat body. J. Biol. Chem. 280, 22624-22631.

Pospisilik, J. A., Schramek, D., Schnidar, H., Cronin, S. J., Nehme, N. T., Zhang, X. Knauf, C., Cani, P. D., Aumayr, K., Todoric, J. et al. (2010). Drosophila genomewide obesity screen reveals hedgehog as a determinant of brown versus white adipose cell fate. Cell 140, 148-160.

Schlegel, A. and Stainier, D. Y. (2007). Lessons from "lower" organisms: what worms, flies, and zebrafish can teach us about human energy metabolism. PLoS Genet. 3, e199.

Singh, R., Kaushik, S., Wang, Y., Xiang, Y., Novak, I., Komatsu, M., Tanaka, K., Cuervo, A. M. and Czaja, M. J. (2009). Autophagy regulates lipid metabolism. Nature 458, 1131-1135.

Sztalryd, C., Xu, G., Dorward, H., Tansey, J. T., Contreras, J. A., Kimmel, A. R. and Londos, C. (2003). Perilipin A is essential for the translocation of hormonesensitive lipase during lipolytic activation. J. Cell Biol. 161, 1093-1103.

Tansey, J. T., Sztalryd, C., Gruia-Gray, J., Roush, D. L., Zee, J. V., Gavrilova, O. Reitman, M. L., Deng, C. X., Li, C., Kimmel, A. R. et al. (2001). Perilipin ablation results in a lean mouse with aberrant adipocyte lipolysis, enhanced leptin production, and resistance to diet-induced obesity. Proc. Natl. Acad. Sci. USA 98, 6494-6499.
Thiele, C. and Spandl, J. (2008). Cell biology of lipid droplets. Curr. Opin. Cell Biol. 20, 378-385

Tian, Y., Bi, J., Shui, G., Liu, Z., Xiang, Y., Liu, Y., Wenk, M. R., Yang, H. and Huang, X. (2011). Tissue-autonomous function of Drosophila seipin in preventing ectopic lipid droplet formation. PLoS Genet. 7, e1001364.

Walther, T. C. and Farese, R. V., Jr (2009). The life of lipid droplets. Biochim. Biophys. Acta 1791, 459-466.

Welte, M. A., Cermelli, S., Griner, J., Viera, A., Guo, Y., Kim, D. H., Gindhart, J. G. and Gross, S. P. (2005). Regulation of lipid-droplet transport by the perilipin homolog LSD2. Curr. Biol. 15, 1266-1275.

Wolins, N. E., Quaynor, B. K., Skinner, J. R., Schoenfish, M. J., Tzekov, A. and Bickel, P. E. (2005). S3-12, Adipophilin, and TIP47 package lipid in adipocytes. J. Biol. Chem. 280, 19146-19155.

Zimmermann, R., Strauss, J. G., Haemmerle, G., Schoiswohl, G., BirnerGruenberger, R., Riederer, M., Lass, A., Neuberger, G., Eisenhaber, F. Hermetter, A. et al. (2004). Fat mobilization in adipose tissue is promoted by adipose triglyceride lipase. Science 306, 1383-1386.

Zimmermann, R., Lass, A., Haemmerle, G. and Zechner, R. (2009). Fate of fat: the role of adipose triglyceride lipase in lipolysis. Biochim. Biophys. Acta 1791, 494 500 . 